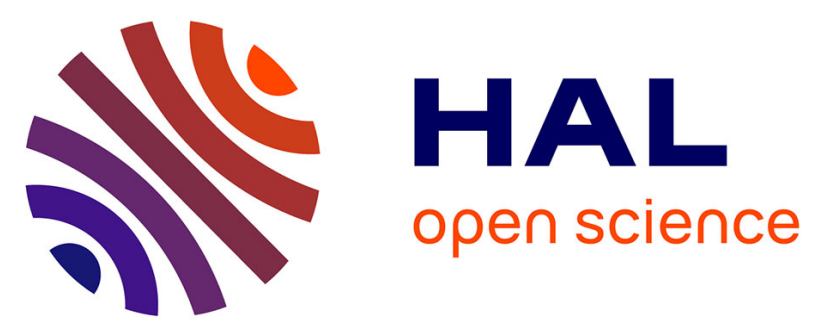

\title{
A Protocol to Quantify Cellular Morphodynamics: From Cell Labelling to Automatic Image Analysis
}

Maria Manich, Aleix Boquet-Pujadas, Stéphane Dallongeville, Nancy Guillén, Jean-Christophe Olivo-Marin

\section{- To cite this version:}

Maria Manich, Aleix Boquet-Pujadas, Stéphane Dallongeville, Nancy Guillén, Jean-Christophe OlivoMarin. A Protocol to Quantify Cellular Morphodynamics: From Cell Labelling to Automatic Image Analysis. Nancy Guillen. Eukaryome Impact on Human Intestine Homeostasis and Mucosal Immunology, Springer International Publishing, pp.351-367, 2020, 978-3-030-44825-7. 10.1007/978-3030-44826-4_25. pasteur-02773115

\section{HAL Id: pasteur-02773115}

https://hal-pasteur.archives-ouvertes.fr/pasteur-02773115

Submitted on 4 Jun 2020

HAL is a multi-disciplinary open access archive for the deposit and dissemination of scientific research documents, whether they are published or not. The documents may come from teaching and research institutions in France or abroad, or from public or private research centers.
L'archive ouverte pluridisciplinaire HAL, est destinée au dépôt et à la diffusion de documents scientifiques de niveau recherche, publiés ou non, émanant des établissements d'enseignement et de recherche français ou étrangers, des laboratoires publics ou privés. 


\title{
Chapter 25
}

\section{A Protocol to Quantify Cellular Morphodynamics: From Cell Labelling to Automatic Image Analysis}

\author{
Maria Manich, Aleix Boquet-Pujadas, Stéphane Dallongeville, \\ Nancy Guillén, and Jean-Christophe Olivo-Marin
}

\begin{abstract}
Cellular morphodynamics can be used as markers for many physiological and pathological processes. This protocol provides a step-by-step guide to identify variations in motility and morphology within (or across) cell populations using non-invasive live imaging and reproducible image analysis techniques such as segmentation and tracking. Detailed instructions cover all the way from cell culturing and labelling to automatic image and statistical analyses, including the definition of multiple descriptors that characterise the shape and movement of cells in a quantitative manner. All methods are available as free open-source software and illustrated by video tutorials.
\end{abstract}

Keywords Cell biology ' Image analysis · Software - Segmentation • Tracking • Shape descriptors

Electronic supplementary material The online version of this chapter (https://doi.org/10.1007/978-3-030-44826-4_25) contains supplementary material, which is available to authorized users.

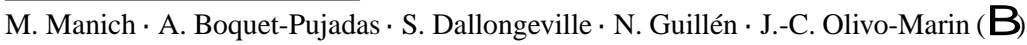
Institut Pasteur, Bioimage Analysis Unit, 75015 Paris, France e-mail: jcolivo@pasteur.fr

M. Manich · A. Boquet-Pujadas · S. Dallongeville · J.-C. Olivo-Marin Centre National de La Recherche Scientifique, CNRS, UMR3691 Paris, France

\author{
A. Boquet-Pujadas \\ Sorbonne Université, Paris, France \\ N. Guillén \\ Centre National de La Recherche Scientifique, CNRS, ERL9195 Paris, France




\section{Introduction}

Advances in microscopy techniques and fluorescent probes have long being helping the scientific community determine the importance of cell movement and deformation in multiple biological processes. However, many studies remain qualitative, i.e. differences in shape or motility are assessed visually, adding subjectivity to potential biological conclusions. Conversely, using image analysis to assign numerical values to both shape and movement does not only guarantee the reproducibility of the conclusions but also opens the door to statistical analyses that allow classifying cell populations and phenotyping. Accordingly, we present a step-by-step manual that shows how to quantify cellular morphodynamics in a non-invasive and reproducible way using only confocal microscopy and fluorescent markers.

The present protocol details both biological and computational experiments. We first describe the necessary biological techniques, namely culturing the cells and fluorescently labelling the cytoplasm; next, we comment on how to perform non-invasive imaging using a confocal microscope; and, finally, we provide a ready-to-use image analysis workflow that goes all the way from raw images to biological conclusions in a reproducible manner. More specifically, we present automatic tools for cell segmentation and tracking that are freely accessible as modules in the Icy platform; as well as multiple descriptors that quantify cell shape and movement from the resulting contours and tracks. These descriptors serve as a basis from which to perform statistical tests and assess any possible correlation between morphodynamical variables. All the key steps of the protocol are available as video tutorials and are exemplified using a population of Entamoeba histolytica, a highly motile parasite that migrates through diverse human tissues, including the intestine and the liver.

\section{Results}

\section{Wet-Lab Protocol: Culturing Cells and Acquiring Images}

To quantify movement and deformation using image analysis (see dry-lab protocol), it is paramount to image the cells non-invasively (physiological relevance) and in good spatiotemporal resolution (easier analysis). To meet these two criteria, we label the cytoplasm with a fluorescent dye and use a spinning-disk confocal microscope.

\section{Cell Culture and Staining}

Trophozoites of the Entamoeba histolytica strain HM1: IMSS were grown overnight at $37{ }^{\circ} \mathrm{C}$ in TYI-S-33 medium (Diamond et al. 1978). Medium was then replaced by incomplete TYI-S-33 medium (serum/vitamines-free) (TYI). Cells were labeled with Cell TrackerTM Red CMTPX, a fluorescent dye that is well suited for monitoring 
cell movement and displacements (Petropolis et al. 2014). The dye has low cytotoxicity, does not affect cell viability nor proliferation, and its fluorescence was stable during the entire imaging process, allowing us to track cellular movements with a red excitation/emission spectra $(577 / 602 \mathrm{~nm})$. In this case, we have used the fluorescent dye that emits in the red spectra because Entamoeba histolytica autofluoresces at $488 \mathrm{~nm}$. Since the forthcoming image analysis methods are based on accurate cell segmentation, in this paper we used non-confluent cultures. Other image analysis tools are required to deal with confluent cell cultures, but they are not the focus of this paper.

Cells were incubated for $45 \mathrm{~min}$ at $37^{\circ} \mathrm{C}$, and then washed with TYI pre-warmed at $37^{\circ} \mathrm{C}$ by reversing the tube and simply discarding the medium. No centrifugation is required because amoebas are adherent cells and remain attached to the glass tube during the process. Trophozoites were gently suspended in pre-warmed TYI by shaking the tube and then seeded on $35 \mathrm{~mm}$ glass-bottomed imaging Ibidi dishes, obtaining an estimate of $5 \times 10^{3}$ cells.

\section{Microscopy Experiment}

Images were taken with a spinning disk confocal microscope $(25 \times$ objective $)$ inside an incubator at $37^{\circ} \mathrm{C}$ to keep the parasites at a physiological temperature where they are specially motile. Indeed, at these temperature E. histolytica parasites can move at up to $1 \mu \mathrm{m} / \mathrm{s}$ in 2D culture conditions (Dufour et al. 2015). Fortunately, with the spinning disk microscope, images can be acquired at very high frame rates with minimal illumination and photo-bleaching of the living samples.

Videos were recorded for four minutes at an imaging rate of one frame per second (i.e. a total of 240 frames) and at a pixel size of $0.48 \mu \mathrm{m}$. The fields of view were taken to be of around $512 \times 512$ pixels, corresponding to $246 \times 246 \mu \mathrm{m}^{2}$, which typically contained around 2-6 cells. The $z$ position was set at a height of around $2 \mu \mathrm{m}$ from the glass.

Both pixel size and frame rate are necessary for the posterior image analysis, for example to obtain the speed in real units, and therefore need to be stored. They are typically stored automatically in the metadata of the image files by the software associated with the microscope, but we recommend to double-check that this is indeed the case. In our case, all images were acquired with the Volocity 3D image analysis software (Perkin Elmer, USA) and the files and their associated metadata stored in the $\operatorname{mvd} 2$ format.

There are no potential dangers involved in the experiments, neither because of laser beams nor of parasite pathogenicity. However, a P2-class laboratory is needed to handle the living trophozoites. The protocol was set up according to the guidelines provided by the Safety Authorities and the Image Microscopy Facility platform of Institut Pasteur. 


\section{Dry-Lab Protocol: Analysing Images}

The motility of a cell population can be studied quantitatively using image analysis. In this context, each individual cell in a video sequence is first singled out of the background in a process called segmentation. Cell segmentation not only allows to delimit the borders of the cells present in an image, but also to calculate their centroid and thus to track the displacement of the cells over time. On the one hand, digitising the contours of the cell opens the door to characterising the cell shape with descriptors such as roundness; on the other hand, the time tracks contain information on the movement of the cell such as its speed or the straightness of its trajectory, which shed light on the reasons behind its migration (random, directed chemotaxis, etc.). Therefore, these data enable a rich quantification of both cell morphology and motility that ideally translates into cell phenotyping when complemented with an extensive statistical and correlation analysis.

The three main steps (segment, track and statistical assessment) are visited in detail in respective Sects. "Hierarchical K-Means", "Active Contours", "Cell Tracking with Track Manager" and are automatised by bioimaging softwares such as Icy (de Chaumont et al. 2012; Wiesmann et al. 2015), which we address immediately in Sect. 25.2.2. All steps are available as video tutorials.

\section{Bioimage Analysis Software}

To quantify cell motility, we present Icy, a free and open-source platform for bioimage analysis that provides multiple resources to visualize, annotate and quantify bioimaging data (http://icy.bioimageanalysis.org).

Icy provides a user-friendly approach to new and classical image analysis techniques alike: filtering, segmentation, tracking.... They are all available under different modules called plug-ins who all share the same graphical interface. Examples of segmentation plug-ins are Thresholding, Active Contours, Parametric Snakes, Potts Segmentation, Spot Detector (Olivo-Marin 2002) and HK-Means (Dufour et al. 2008); whereas plug-ins such as Spot Tracking, Track Manager and Kymograph Tracker provide different approaches to tracking. In this protocol, we will focus on HKMeans (Sect. "Hierarchical K-Means"), Active Contours (Sect. "Active Contours"), and Track Manager (Sect. "Cell Tracking with Track Manager") in order to provide a step-by-step guide on how to analyse cell shape and motility.

The graphical interface integrates $2 \mathrm{D}$ and $3 \mathrm{D}$ visualisation resources, as well as a series of tools to easily crop and cut through time series, z-stacks or multichannel sequences. Also intuitive is the management of so-called Regions Of Interest (ROIs), i.e. delimited areas of the image that are of special interest and that might want to be analysed aside, for example a cell segmented from the image. In the Icy platform, ROIs are superimposed over the original image and can be manipulated as independent objects on which common operations such as "copy/paste" (ctrl + $\mathrm{c} / \mathrm{v}$ ) or "delete" can be applied, allowing to easily combine analyses performed on 
different channels or sequences. ROIs are deeply integrated into Icy so that any analysis or quantification from them is automatic and straightforward. For instance, cell segmentation results are represented as ROIs from which multiple descriptors such as area, mean fluorescence intensity or roundness can be directly accessed in the ROI menu and exported into Excel files for further data analysis.

As a last remark, in its most recent version 2.0, Icy has introduced a new image handling engine that allows working with big video sequences, be it either because they are long or because they were taken at very high spatial and/or temporal resolution. The idea behind the new engine is that only a portion of the image sequence is loaded into the local RAM memory, while the rest is stored on hard-disk at the price of longer processing times.

We have used the sequence called $25 \times 40$ to illustrate this protocol (Movie 1) over its several steps. The Time Stamp Overlay plugin (Tutorial 1) can be used to stamp the elapsed time onto the video.

\section{Cell Segmentation with Hierarchical K-Means and Active Contours}

\section{Hierarchical K-Means}

Hierarchical K-Means (HK-means or HKM here) is a segmentation method based on a K-Means clustering of the image histogram, i.e. an algorithm that divides the different intensity values in the image into groups according to a similarity measure. Ideally, these groups correspond to the different cells and to the background. However, the K-means algorithm requires the number of groups to be specified in advance. To tackle this problem, a hierarchical strategy is introduced. In this way, the algorithm attempts to find the ideal number of groups using a bottom-up approach. This process can be helped if the user specifies a value for the expected minimum and maximum size of the cells.

HKM is a fundamental tool in image processing; it is one of the go-to algorithms if the user wants to segment cells in a quick, ready-to-use and quasi-automatic manner. And precisely because of its hierarchical clustering approach, it performs better than classical clustering and thresholding algorithms. However, HKM suffers from three main drawbacks. First, it has difficulty telling apart cells that are in contact with each other. Second, big intensity heterogeneities inside the cell might trigger multiple detections. And third, since the resulting segmentations are groups of pixels rather than polygonal contours, some accuracy may be lost when computing shape descriptors.

A step-by-step guide to the HKM plug-in in Icy can be found in Fig. 25.1 and is accompanied by Tutorial 2 . 

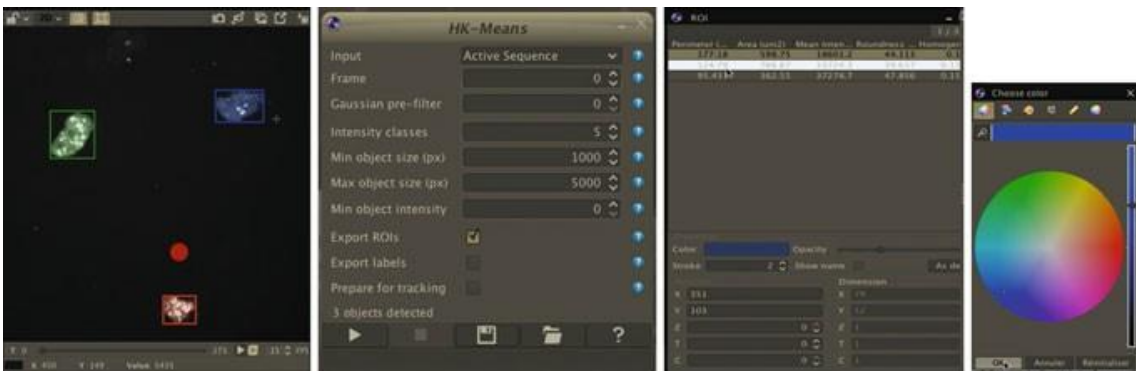

Fig. 25.1 Cell segmentation with HK-Means and ROI color selection. HK-Means can be opened from the detection tab or from the search bar. The first step is to choose which frames we are interested in segmenting (here we select frame 0 , but 'ALL' is also a possibility). We have to specify the number of intensity classes (see the text for an explanation on HK-Means) in which the histogram is to be split. That is the number of different intercellular intensities (e.g. if all cells are the same intensity, two classes are enough). When in doubt, we recommend choosing higher values, but we also remark that it comes at a computational price. To aid the segmentation of the image into the different classes the user can also input an expected minimum and maximum size for the cells so as to eliminate possible groups that are respectively too small (e.g. debris) or too big to possibly be a cell (e.g. cell clusters). Notice that these sizes are required in pixels, to have a rough idea of the cell size in pixels draw a ROI around the cell and check its size ("Interior" descriptor in the ROI tab at the right-hand side). Finally, applying the Gaussian pre-filter can help improve the segmentation of noisy images. Since the segmentation output are ROIs, we can obtain any descriptor directly from the ROI tab. Here we show the perimeter, the area, the mean intensity, the roundness and the homogeneity inside the ROIs, but many more shape descriptors can be selected using the "gear" button. ROI colors can be chosen (see Tutorial 2)

\section{Active Contours}

Active Contours (Zimmer et al. 2002) (AC) are well adapted to study cell morphodynamics; they provide accurate cell contours and are capable of segmenting cells that are in close contact, as well as cells with inner heterogeneities. However, in contrast to the more classical segmentation methods, AC need to be initialised. The user has to specify an approximative initial contour (ROI) around the cell so that the algorithm can pick up on it. This initialization can be done manually, by drawing the ROI over the object of interest, or automatically, using other segmentation tools (e.g. the above-described HKM). The initial ROI contours are then refined by the AC method, which slowly deforms the contour. In this way, the contour is progressively fitted to the cell shape in an attempt not only to separate the image into multiple intensities, but also to find the edges of the cells in the gradient of the image. When the segmentation spans a whole video sequence, the ROI resulting from segmenting a given frame can be used as an initial ROI for the following frame (see 'track objects over time' in the AC plug-in). Therefore, if the image acquisition is relatively fast, initialising the ROIs at the very first frame is enough to segment the entire sequence.

In summary, whereas HKM is fast and does not need to be initialised, it is most performant when image quality is good and cells are well separated; otherwise AC take over at the price of initialisation and speed. In fact, we remark that a good 
a

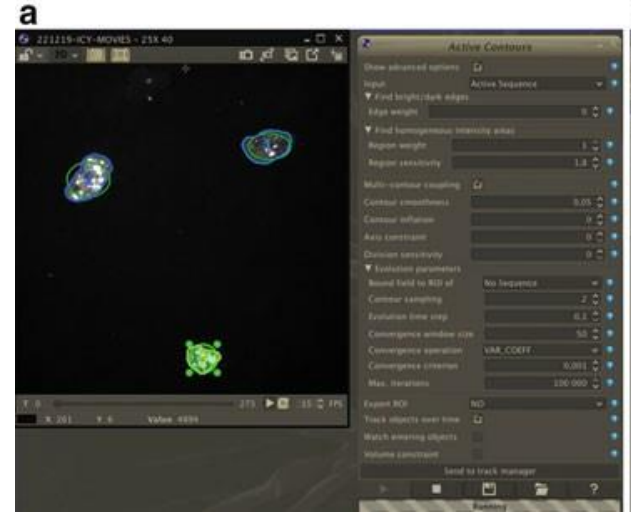

b

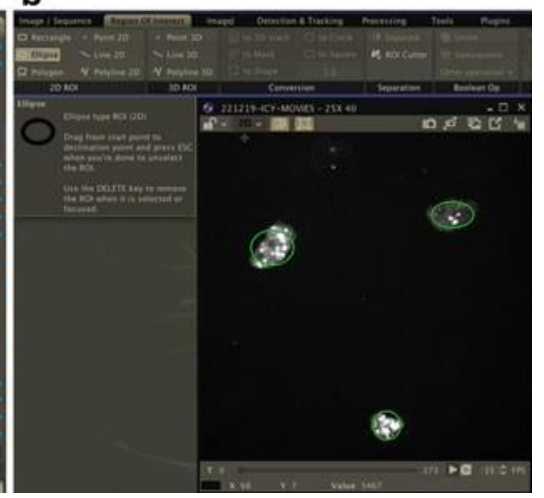

Fig. 25.2 Manual drawing of approximative ROIs and automatic cell segmentation with Active Contours. a The first step before running Active Contours is to draw approximative ROIs around each of the cells; we do it manually here (green ellipses), but one could use HK-Means to initialize the method automatically. b Active Contours can be opened from the detection tab or from the search bar. In order to segment and track all of the time frames make sure to activate "tracks objects over time". Perhaps the two most important parameters of the plug-in are the edge and region weights, which control the balance between the importance of (1) the big intensity differences that are expected at the border of the cell and (2) the homogeneous intensity that is expected inside the cell as opposed to that of the outside. In addition, "contour inflation" might help compensate for a lack of contrast between cell and background by adding an artificial expansion rate. On the other hand, the set of evolution parameters are more technical but can help speed up the process and/or make the final contours more accurate. All parameter settings can be readily stored and loaded using the save icon on the bottom. The results of the segmentation are also presented as ROIs (see text and Tutorial 3)

approach is to combine the two; that is to use HKM (only) on the first frame to automatically set the initial ROIs required by AC. However, in this protocol we have found it more pedagogic to set the initial contours manually.

A step by step guide to the AC plug-in in Icy can be found in Fig. 25.2 and is accompanied by Tutorials 2, 3 and 4. Movie 2 shows the segmented cells with Active Contours.

\section{Cell Tracking with Track Manager}

Using either of the segmentation plug-ins on a video sequence results in a time-series of ROIs that can potentially be linked together to generate the track of a cell, i.e. to draw the path that the cell followed. At our spatiotemporal resolution, it suffices to associate a ROI at a given time point with the closest ROI at the following time point to accurately track cells; more precisely, it is the centroids of the successive ROIs that are concatenated into a cell track. However, more advanced tracking tools such as Multiple Hypothesis Tracking (Chenouard et al. 2013) become necessary for high-speed particle tracking. In either case, the resulting tracks can be analyzed 


\section{a)}

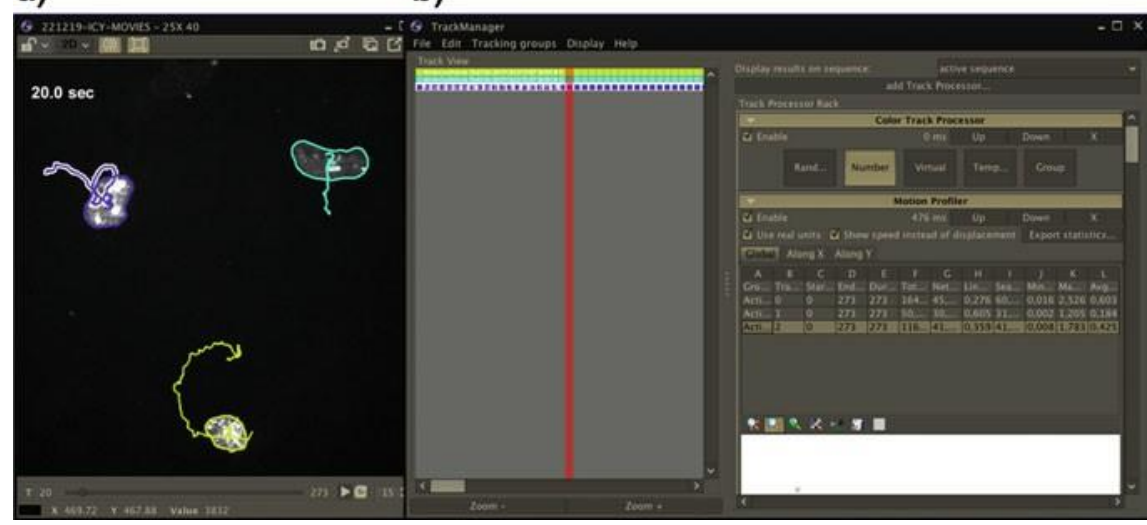

with the Track Manager (TM) module in Icy, which is readily invoked from the very segmentation plug-ins using the 'send to track manager' button.

TM displays the resulting tracks directly overlayed on the original sequence. The tracks can also be analyzed through an accessible interface, for instance to investigate motility parameters such as cell speed or mean squared displacement (MSD) and compare them between populations or correlate them with other descriptors, for example of cell morphology (see below). All these quantifications tasks are done through so called Track Processors (TPs). Each TP has a specific function: from filtering unwanted tracks, to quantifying movement, passing by a myriad of display functionality such as color-coding the tracks ("TP Color"). In this protocol, we have used several TPs. Briefly, (i) "Motion Profiler" computes multiple motion descriptors such as the average speed or the linearity/persistence of the tracks; (ii) "Instant Speed" displays the speed of the cell as a time curve; whereas (iii) "ROI Statistics" (ROIS) displays time curves of several shape descriptors as is described in Sect. "Statistical Tests with R".

A step-by-step guide to the TM plug-in in Icy can be found in Figs. 25.3, 25.4 and 25.5 and is accompanied by Tutorial 5 . Movie 3 shows the segmented cells with Active Contours and their centroid tracks.

Fig. 25.3 Cell track analysis with Track Manager and Track Processors. a Track Manager can be opened from the tabs or directly from most segmentation plug-ins (e.g. HK-Means or Active Contours, see respective Figs. 25.1, 25.2 and Tutorial 4). The tracks for each of the cells are automatically overlaid on the video sequence in the corresponding colors. b Each track is a separate entity and can be filtered or quantified by adding Track Processors. Here we are displaying the Color and Instant Speed Track Processors, but many others are available (e.g. see Figs. 25.4, 25.5 and 25.6). The red vertical bar displays the current time point, and can be dragged to navigate the time sequence. Tracks can be saved into an.xml file 
a)

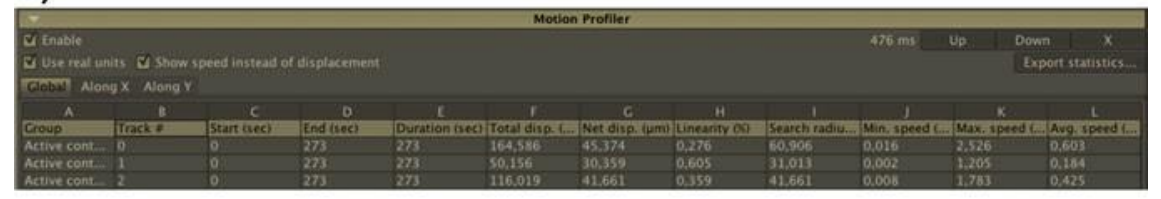

b)

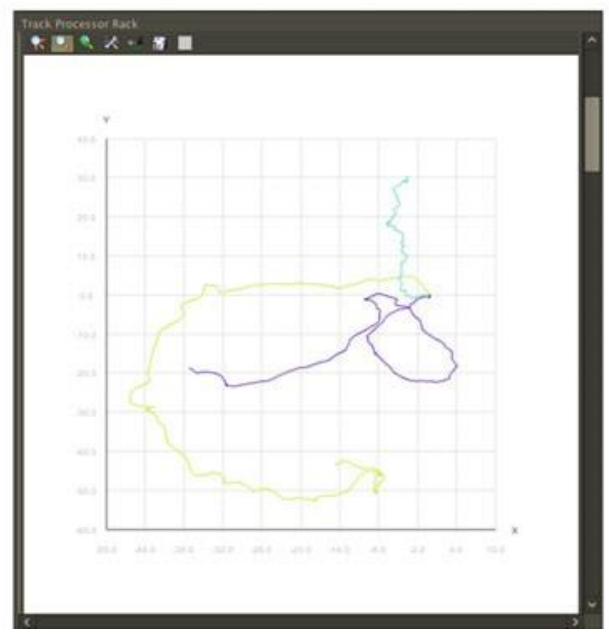

Fig. 25.4 Descriptors and visualisation of cell tracks with the Motion Profiler Track Processor in Track Manager. a By selecting Motion Profiler from the track processors in Track Manager, we obtain multiple descriptors of the three tracks. For example, we can see typical minimum, maximum and average speed values; and we can also quantify how straight the cells are moving with the measures of linearity and search radius. In addition, the processor can take the metadata into account to offer the values in real units. All these results can be exported to an Excel file. b Motion Profiler also provides a graphical representation of all the tracks from a common origin, from where we can visually assess whether motion is random or directed

\section{Morphological Descriptors and Statistical Tests with ROI Statistics and R}

\section{Cell Descriptors with ROI Statistics}

Different cell populations might be characterised by different morphologies. Given a time sequence of already segmented cells in the form of ROIs (e.g. with AC), the ROI

Statistics (ROIS) processor in TM provides a wide range of geometrical properties that describe the shape of each ROI. Together with a posterior statistical analysis, these descriptors may help tell apart different populations or be used for phenotyping. Many such descriptors are available in ROIS; in this study, we only consider the fol- 


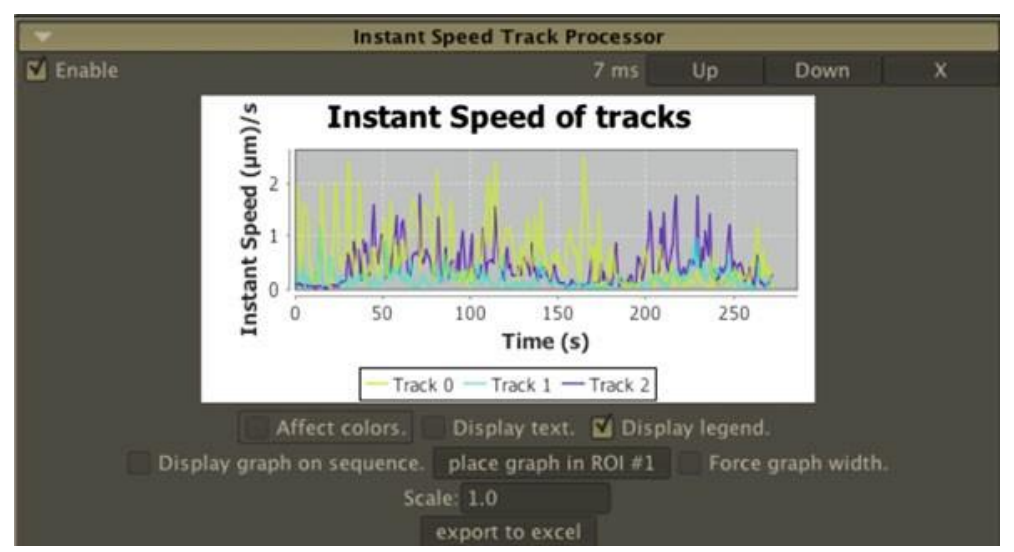

Fig. 25.5 Time curves of cell speed with the Instant Speed Track Processor in Track Manager. By selecting Instant Speed from the track processors in Track Manager, we obtain the time curves of the speed at each time point along each of the cell tracks. These data can be used to explore the cycles of acceleration and deceleration of the cells for example. The curves can be exported to an Excel file

lowing: area $\left(\mu \mathrm{m}^{2}\right)$, perimeter $(\mu \mathrm{m})$, roundness $(\%)$, mean intensity values (a.u.) and homogeneity (a.u.). "Perimeter" measures the perimeter of the ROI in micrometers (here the scaling information is extracted automatically from the metadata). Equivalently, "Area" measures the ROI area in micrometers squared. "Mean intensity" averages the intensity values inside the ROI, whereas "Homogeneity" highlights the internal variations of the intensity distribution within the cell. Lastly, "Roundness" is a measure of how similar to a circle the ROI is. These data are displayed directly in Icy, but can also be exported to an Excel file (Table 25.1) for further analysis, for example to perform statistical tests that assess the correlation between each of the descriptors. For instance, we study the correlations between the temporal mean of all these parameters and the Speed $(\mu \mathrm{m} / \mathrm{s})$ resulting from TM. Alternative shape descriptors can be extracted by rewriting the cell shape in different mathematical basis such as Fourier (2D) or Spherical Harmonics (3D); these work well to separate populations, but often lack biological interpretability (Ducroz et al. 2012).

A step-by-step guide to the TM plug-in in Icy can be found in Fig. 25.6.

\section{Statistical Tests with R}

In order to assess whether any trend or correlation exists between the extracted descriptors we perform a visual pairwise comparison educated with Spearman's rank correlation coefficient. So-called Spearman's "rho" attempts to quantify the monotonicity of the relationship between a pair of variables, irrespectively of its linearity. The coefficient spans the interval $[-1,1]$, where the extremes correspond to perfectly monotonic functions, respectively decreasing or increasing (i.e. functions 


\begin{tabular}{|c|c|c|c|c|c|c|}
\hline \multirow[t]{2}{*}{ Name } & Area & Homogeneity & Intensity & Perimeter & Roundness & Speed \\
\hline & $(\mu \mathrm{m} 2)$ & $(561)$ & $(561)$ & $(\mu \mathrm{m})$ & $(\%)$ & $(\mu \mathrm{m} / \mathrm{s})$ \\
\hline \multirow[t]{2}{*}{$25 \times 54$} & 316.08 & 0.08 & 24,203 & 75.58 & 40.05 & 1.16 \\
\hline & 2407.56 & 0.12 & 19,189 & 196.18 & 59.33 & 0.08 \\
\hline \multirow[t]{2}{*}{$25 \times 49$} & 2779.13 & 0.13 & 24,801 & 208.46 & 59.11 & 0.24 \\
\hline & 308.78 & 0.15 & 15,070 & 69.42 & 48.16 & 1.21 \\
\hline \multirow{2}{*}{$25 \times 47$} & 771.28 & 0.13 & 16,320 & 119.90 & 34.82 & 0.17 \\
\hline & 411.95 & 0.13 & 21,341 & 81.62 & 49.3 & 0.43 \\
\hline \multirow{6}{*}{$25 \times 44$} & 760.11 & 0.10 & 25,160 & 114.79 & 45.55 & 0.40 \\
\hline & 684.88 & 0.10 & 25,544 & 100.88 & 68.20 & 0.12 \\
\hline & 545.58 & 0.11 & 21,754 & 90.64 & 58.77 & 0.97 \\
\hline & 689.35 & 0.10 & 23,625 & 108.82 & 48.97 & 0.25 \\
\hline & 658.80 & 0.10 & 23,308 & 109.13 & 46.66 & 0.49 \\
\hline & 929.79 & 0.10 & 23,857 & 119.42 & 61.02 & 0.16 \\
\hline \multirow[t]{3}{*}{$25 \times 40$} & 713.25 & 0.18 & 42,758 & 109.02 & 54.33 & 0.42 \\
\hline & 438.46 & 0.32 & 46,123 & 88.07 & 42.48 & 0.60 \\
\hline & 773.17 & 0.15 & 17,600 & 118.27 & 36.11 & 0.18 \\
\hline \multirow[t]{3}{*}{$25 \times 38$} & 362.51 & 0.11 & 18,333 & 73.27 & 59.32 & 1.05 \\
\hline & 1045.82 & 0.10 & 22,956 & 132.80 & 37.29 & 0.10 \\
\hline & 814.01 & 0.10 & 21,460 & 113.92 & 54.76 & 0.33 \\
\hline \multirow[t]{4}{*}{$25 \times 36$} & 1093.23 & 0.10 & 21,429 & 135.41 & 52.52 & 0.14 \\
\hline & 525.07 & 0.08 & 27,100 & 92.00 & 50.12 & 0.31 \\
\hline & 2713.55 & 0.10 & 36,252 & 233.99 & 29.84 & 0.10 \\
\hline & 600.94 & 0.11 & 18,237 & 95.02 & 60.56 & 0.61 \\
\hline \multirow[t]{5}{*}{$25 \times 35$} & 329.77 & 0.1 & 22,166 & 73.21 & 47.95 & 1.10 \\
\hline & 630.77 & 0.1 & 24,413 & 101.25 & 51.82 & 1.37 \\
\hline & 681.54 & 0.1 & 21,612 & 107.25 & 48.32 & 0.14 \\
\hline & 466.57 & 0.1 & 33,879 & 85.38 & 53.27 & 0.23 \\
\hline & 617.05 & 0.11 & 19,998 & 104.93 & 41.30 & 0.22 \\
\hline \multirow[t]{2}{*}{$25 \times 34$} & 656.30 & 0.34 & 49,554 & 108.70 & 43.97 & 0.32 \\
\hline & 663.35 & 0.21 & 10,132 & 103.12 & 47.39 & 0.29 \\
\hline \multirow[t]{2}{*}{$25 \times 33$} & 207.05 & 0.07 & 24,868 & 56.05 & 52.87 & 0.62 \\
\hline & 595.10 & 0.13 & 16,759 & 97.52 & 52.17 & 0.86 \\
\hline \multirow[t]{2}{*}{$25 \times 32$} & 523.20 & 0.13 & 16,121 & 94.08 & 45.30 & 0.54 \\
\hline & 653.94 & 0.11 & 19,977 & 105.08 & 41.45 & 0.14 \\
\hline $25 \times 30$ & 1084.28 & 0.11 & 23,447 & 130.04 & 30.10 & 0.15 \\
\hline
\end{tabular}

Table 25.1 Cell shape descriptors and speed for the $\mathbf{4 2}$ cells of the experiment. $(25 \times 40$ illustrates the protocol, values are means) 
Table 25.1 (continued)

\begin{tabular}{l|l|l|l|l|l|l}
\hline \multirow{2}{*}{ Name } & Area & Homogeneity & Intensity & Perimeter & Roundness & Speed \\
\cline { 2 - 7 } & $(\mu \mathrm{m} 2)$ & $(561)$ & $(561)$ & $(\mu \mathrm{m})$ & $(\%)$ & $(\mu \mathrm{m} / \mathrm{s})$ \\
\hline \multirow{7}{*}{$25 \times 29$} & 887.42 & 0.09 & 23,499 & 128.19 & 39.44 & 0.24 \\
\cline { 2 - 7 } & 738.61 & 0.12 & 17,261 & 107.66 & 51.55 & 0.30 \\
\cline { 2 - 7 } & 791.83 & 0.08 & 26,903 & 115.63 & 47.52 & 0.15 \\
\cline { 2 - 7 } & 515.45 & 0.10 & 20,549 & 85.64 & 70.17 & 0.12 \\
\cline { 2 - 7 } & 430.99 & 0.09 & 23,945 & 90.56 & 35.33 & 0.54 \\
\cline { 2 - 7 } & 532.69 & 0.12 & 17,155 & 93.70 & 49.06 & 0.47 \\
\cline { 2 - 7 } & 437.21 & 0.12 & 16,415 & 85.08 & 47.43 & 0.37 \\
\cline { 2 - 7 } & 799.27 & 0.10 & 22,078 & 118.07 & 42.83 & 0.64 \\
\hline
\end{tabular}

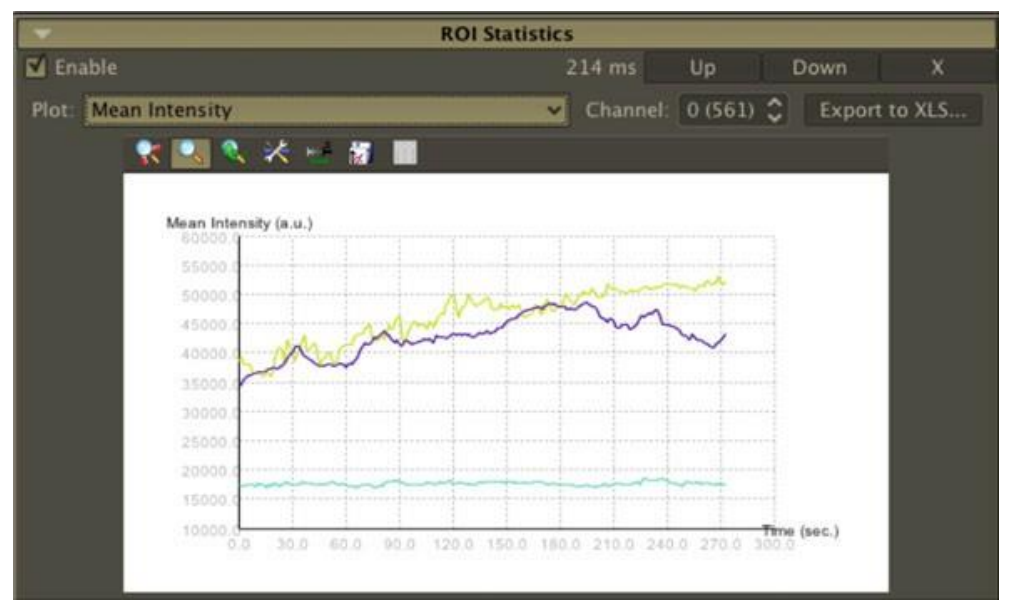

Fig. 25.6 Cell shape descriptors with the ROI Statistics Track Processor in Track Manager. By selecting ROI Statistics from the track processors in Track Manager, we obtain different descriptors of cell shape (perimeter, roundness, etc.) for each time point along a cell track. Here are presented the fluorescence average intensity values inside the ROIs. The data can be exported to an Excel file

that always go down, or up, without fluctuations); and 0 indicates a lack of correlation. Precisely, the p-value associated with the coefficient results from testing whether this coefficient is significantly different from 0 .

Statistical analysis software can directly read the output values exported from Icy. Here, we use a short $\mathrm{R}$ routine that can automatically generate the pairwise graphics showing possible trends, as well as the correlation values and their corresponding p-values (Fig. 25.7). This program uses some functionality from the ggplot2 library. While it is not the aim of the paper to provide in-depth statistical insight, we remark that it is important to check whether your data satisfies all the assumptions made during the statistical analysis. For illustrative purposes, in Fig. 25.7 generated by 


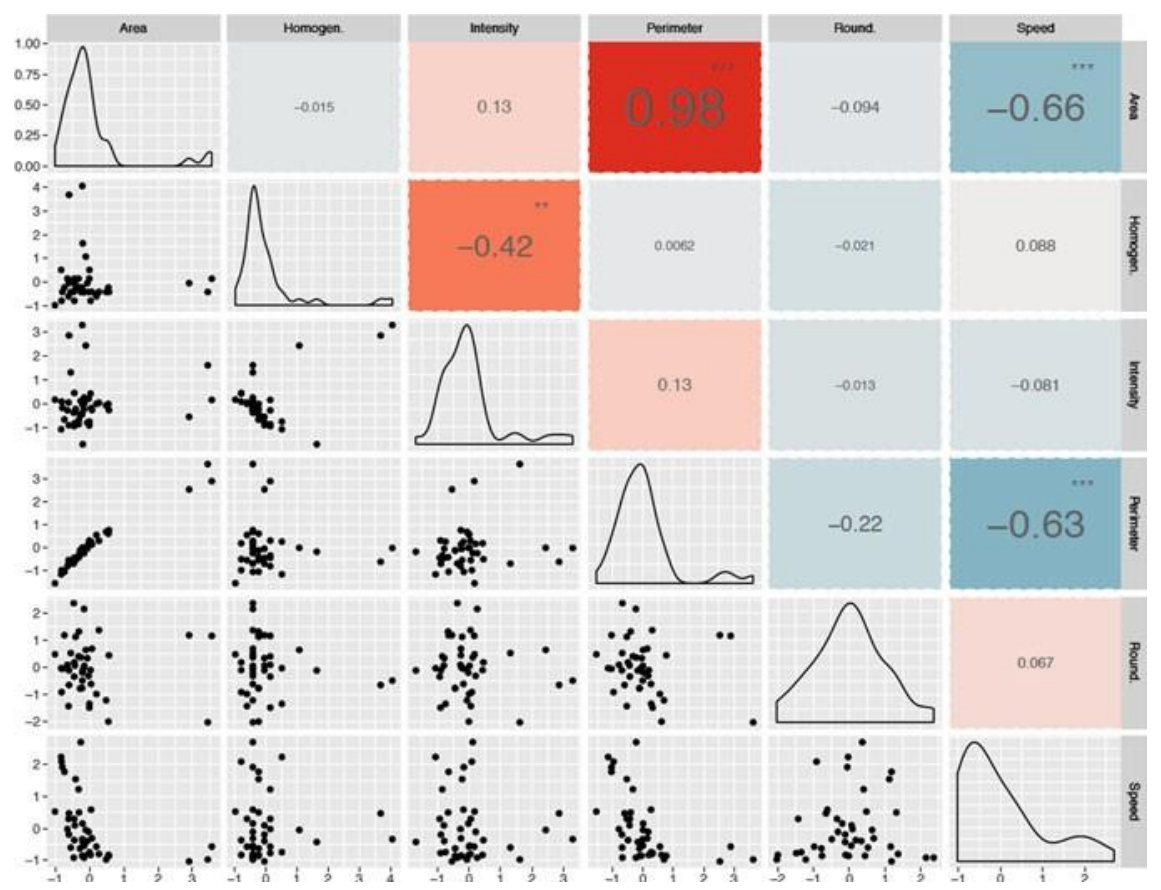

Fig. 25.7 Statistical analysis of cell descriptors shows a significant correlation between cell speed and size. The figure is a matrix quantifying the correlation between pairs of descriptors for $\mathrm{n}=42$ cells from 13 different movies. On the upper triangular side, Spearman's correlation values resulting from descriptor pairs are displayed accompanied by their significance in the form of stars. The values are also displayed using a color gradient (red positive, blue negative) to facilitate the analysis. On the lower triangular side, we plot descriptor pairs on a normalised scale to show any possible trend. The diagonal contains univariate density plots

the $\mathrm{R}$ routine, we display univariate descriptor density plots (diagonal) and pairwise descriptor plots (lower diagonal), but directly compute the pairwise correlation coefficients and their associated tests (upper diagonal) with no prior analysis.

\section{Example to Illustrate the Proposed Protocol for Image Analysis}

During the in vitro growth of $E$. histolytica, it is common to observe diverse phenotypes regarding the size of the cells, their mobility, the heterogeneity of fluorescence during labeling, etc. We wondered whether the protocol proposed here could help us identify any correlations between these phenotypes. After acquisition of videomicroscopies of $E$. histolytica seeded on glass, the image analysis was performed on $\mathrm{n}=42$ cells from 13 different video sequences. The data highlights several relation- 
ships: the obvious correlation between area and perimeter, a less evident correlation between cell fluorescence intensity and homogeneity (as the image saturates), and a strong and significant (***) correlation between the size of the cell and its mean speed (Table 25.1 and Fig. 25.7). For instance, the five smallest cells moved at $62 \pm$ $14 \mu \mathrm{m} / \mathrm{min}$, whereas the five largest cells moved at $9 \pm 4 \mu \mathrm{m} / \mathrm{min}$. Therefore, this experiment allows to conclude that the smaller cells have a higher average speed in the amoeba population moving on glass. This original observation opens the door to further studies on the molecular mechanisms sustaining the correlation between size and speed of $E$. histolytica when moving on a planar and neutral surface such as glass.

\section{Conclusion}

We expect this protocol to serve as a beginner's guide for cell biologists that would like to capture the morphodynamical characteristics of their live cell populations in a quantitative manner by using image analysis. The results are any potential correlations between multiple morphodynamical descriptors (in the present case, we found a link between cell size and speed), as well as the possible discovery of criteria that can tell apart subpopulations of cells.

\section{Materials and Basic Methods}

\section{Biological Materials}

- Trophozoites of Entamoeba histolytica strain HM1:IMSS growing in TYI-S33 media (Diamond et al. 1978).

- Cell Tracker ${ }^{\mathrm{TM}}$ Red CMTPX (ThermoFisher, catalog number C34554, final concentration $2.5 \mu \mathrm{M})$. Before use, suspend the dessicated dye $(50 \mu \mathrm{g})$ in $8.33 \mu \mathrm{l}$ of DMSO to obtain a $10 \mathrm{mM}$ stock solution. An intermediate dilution (1/200) has to be prepared to avoid aggregates of DMSO and Cell Tracker in the media.

- 35 mm high glass-bottom Ibidi dish (catalog number 81158, Ibidi, France).

\section{Equipment}

- Microbiological safety station with laminar flow to manipulate the cells; wearing a blouse and gloves is mandatory during the experimental steps.

- Spinning disk confocal microscope (UltraVIEW VoX, Perkin Elmer, USA; excitation: $561 \mathrm{~nm}$; objective: $25 \times$; temperature control set to $37^{\circ} \mathrm{C}$ ). 


\section{Softwares}

- Volocity (Perkin Elmer, USA) to perform imaging.

- Icy (Institut Pasteur, France) to perform image analysis.

\section{Summary of the Protocol}

Procedure - The protocol can be summarised as a general workflow (Fig. 25.8) in the following steps: culture the cells and label them with a fluorescent cytoplasm dye; image the cells with a temporal resolution that is appropriate to the cell movement. Save the video sequences on the hard-disk; open the Icy software and allocate RAM according to the expected image size, open the sequence, and double-check the metadata; draw initial ROIs over the cells and run the Active Contours plug-in; send the resulting segmentation to the Track Manager, use the different track processors to analyse cell movement and shape and export them to Excel; perform statistical and correlation tests on the data, for example using $\mathrm{R}$.

Timing-Cell labelling and preparation takes between one and two hours. Live imaging only involves setting up the sample on the microscope and taking multiple video sequences of around 240 frames (i.e. around $4 \mathrm{~min}$ ). Segmentation and tracking takes a fraction of a second per frame. Statistical analysis takes well under an hour.

Troubleshooting - 1 . Check that the Java version in your computer is compatible with Icy. 2. From within the preferences tab in Icy assign RAM memory to the software according to the potential size of your images. 3. Check that your temporal resolution is adequate: if there are too many frames per second compared to the speed of the cells, remove frames in constant intervals in order to lift some computational burden. 4. All stages of the quantification can be saved in their corresponding formats. For example, image sequences can be saved in.tif, whereas ROIs and tracks are saved in.xml. This guarantees complete reproducibility, as slightly different ROIs can result in slightly different segmentations.

Data availability - All data presented in this protocol (files as.tif,.xml,.avi,.mov) and tutorials are available online (Manich 2020) so that any potential user can reproduce the results by following the protocol.

Acknowledgements We acknowledge Marion Louveaux for advice on image analysis reproducibility and data online availability. We are grateful to the technical unit of BioImagerie Photonique of Institut Pasteur for their help with microscopy experiments. A.B.P. is part of the Pasteur-Paris University (PPU) International Ph.D. Program.

Funding This project has received funding from the European Union's Horizon 2020 research and innovation program under the Marie Skłodowska-Curie grant agreement No 665807, the Institut Carnot Pasteur Microbes \& Sante (ANR 16 CARN0023-01), the Labex IBEID (ANR-10-LABX-62IBEID), France-BioImaging infrastructure (ANR-10-INBS-04) and the program PIA INCEPTION (ANR-16-CONV-0005). 
Grow the cells until a pre-determined confluency

Label the cells with fluorescent cytoplasmic marker

Image the cells with an appropriate microscope, ideally with optical sectioning

Open the Icy software, load the image sequences

Check the image properties (e.g. time, pixel size) and the available RAM Pay special attention to the exposure time and the pixel size

Initialize segmentation with HKM or draw approximative ROIs

Run Active Contours to get the contour of the cells and to track their centroids

Select Processors in the Track Manager Export data on Excel files

Perform statistical tests Interpret the results and raise a biological conclusion
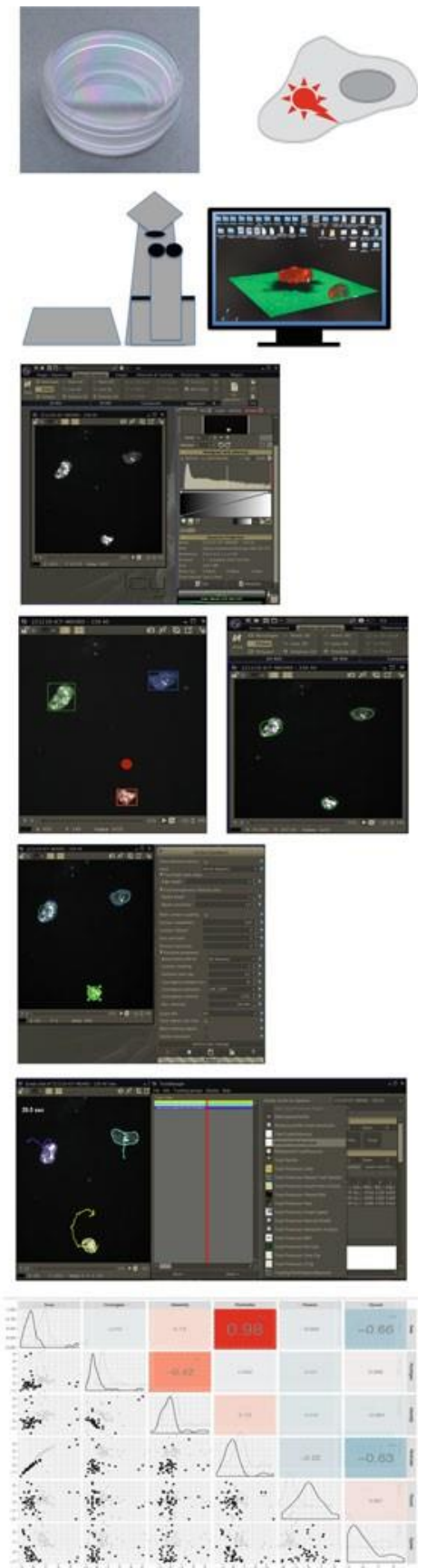

Fig. 25.8 Summary of the protocol. See text for a complete description of the protocole 


\section{References}

Chenouard, N., Bloch, I., \& Olivo-Marin, J. C. (2013). Multiple hypothesis tracking for cluttered biological image sequences. IEEE Transactions on Pattern Analysis and Machine Intelligence, 35(11), 2736-3750. https://doi.org/10.1109/TPAMI.2013.97.

De Chaumont, F., Dallongeville, S., Chenouard, N., Hervé, N., Pop, S., Provoost, T., ... \& Lagache, T. (2012). Icy: an open bioimage informatics platform for extended reproducible research. Nature methods, 9(7), 690.

Diamond, L. S., Harlow, D. R., \& Cunnick, C. C. (1978). A new medium for the axenic cultivation of Entamoeba histolytica and other Entamoeba. Transactions of the Royal Society of Tropical Medicine and Hygiene, 72(4), 431-432. https://doi.org/10.1016/0035-9203(78)90144-X.

Ducroz, C., Olivo-Marin, J. C., \& Dufour, A. (2012). Characterization of cell shape and deformation in 3d using spherical harmonics. In 2012 9th IEEE International Symposium on Biomedical Imaging (ISBI) (pp. 848-851). IEEE. Barcelona. https://doi.org/10.1109/isbi.2012.6235681.

Dufour, A., Meas-Yedid, V., Grassart, A., \& Olivo-Marin, J. C. (2008, December). Automated quantification of cell endocytosis using active contours and wavelets. In 2008 19th International Conference on Pattern Recognition (pp. 1-4). IEEE, Tampa, FL. https://doi.org/10.1109/icpr. 2008.4761748.

Dufour, A. C., Olivo-Marin, J. C., \& Guillén, N. (2015). Amoeboid movement in protozoan pathogens. Seminars in Cell \& Developmental Biology, 46, 128-134. https://doi.org/10.1016/ j.semcdb.2015.10.010.

Manich, M. (2020). Online data of "A protocol to quantify cellular morphodynamics: from cell labelling to automatic image analysis" (Version http://icy.bioimageanalysis.org). Zenodo. https:// doi.org/10.5281/zenodo.3594363.

Olivo-Marin, J. C. (2002). Extraction of spots in biological images using multiscale products. Pattern Recognition, 35, 1989-1996. https://doi.org/10.1016/S0031-3203(01)00127-3.

Petropolis, D. B., Faust, D. M., Deep Jhingan, G., \& Guillen, N. (2014). A New Human 3D-Liver Model Unravels the Role of Galectins in Liver Infection by the Parasite Entamoeba histolytica. PLoS Pathogens, 10(9), e1004381. https://doi.org/10.1371/journal.ppat.1004381.

Wiesmann, V., Franz, D., Held, C., Munzenmayer, C., Palmisano, R., \& Wittenberg, T. (2015). Review of free software tools for image analysis of fluorescence cell micrographs. Journal of Microscopy, 257, 39-53. https://doi.org/10.1111/jmi.12184.

Zimmer, C., Labruyere, E., Meas-Yedid, V., Guillen, N., \& Olivo-Marin, J. C. (2002). Segmentation and tracking of migrating cells in videomicroscopy with parametric active contours: a tool for cell-based drug testing. IEEE Transactions on Medical Imaging, 21(10), 1212-1221. https://doi. org/10.1109/TMI.2002.806292. 